\title{
Les dimensions multiples de l'engagement ludique
}

\section{Olivier Caïra}

\section{(2) OpenEdition}

\section{Journals}

Édition électronique

URL : http://journals.openedition.org/sdj/1149

DOI : $10.4000 /$ sdj. 1149

ISSN : 2269-2657

\section{Éditeur}

Laboratoire EXPERICE - Centre de Recherche Interuniversitaire Expérience Ressources Culturelles Education

\section{Référence électronique}

Olivier Caïra, "Les dimensions multiples de l'engagement ludique ", Sciences du jeu [En ligne],

10 | 2018, mis en ligne le 28 octobre 2018, consulté le 23 avril 2019. URL : http://

journals.openedition.org/sdj/1149; DOI : 10.4000/sdj.1149

Ce document a été généré automatiquement le 23 avril 2019.

Tous droits réservés 


\title{
Les dimensions multiples de l'engagement ludique
}

\author{
Olivier Caïra
}

- C'est mon tour, là ? Alors, je peux faire quoi avec un jaune et un marron?

- Bah, je t'ai expliqué : tu as tous les coûts de construction là, devant toi. Et puis on dit un Or et un Bois, pas un jaune et marron.

- Hé, ça va, on reste cool... On est là pour jouer, non?

- Ouais, justement, si tu étais un minimum au jeu, je resterais cool!

1 Ce bref échange, noté lors d'une partie du jeu de plateau Kingsburg (Chiarvesio et Iennaco, 2007), illustre la notion de multiplicité des formes d'engagement qui peuvent s'observer autour d'une table de jeu. Ce qui m'a intéressé, dans ces quatre répliques, c'est que le premier joueur, à qui sa voisine agacée reproche une forme de sous-engagement dans la partie, répond par un désarmant « On est là pour jouer, non?». Il sous-entend que, dans sa vision du jeu, la convivialité et la détente, l'ambiance « cool» autour de la table sont plus importants que d'autres composantes de l'expérience ludique qu'il néglige tacitement : le système de règles (« C'est mon tour? », « je peux faire quoi ?») et le thème médiéval (« un jaune et un marron» pour « un Or et un Bois»). Ses propos, ainsi que sa simple présence autour de la table, indiquent qu'il est convaincu d'être « au jeu », alors qu'il exaspère manifestement les autres participants.

2 Parmi les multiples interprétations possibles de la notion d'engagement, je m'en tiendrai à la plus neutre, en apparence la plus simple : être engagé dans le jeu et s'engager vis-àvis des joueurs. L'engagement ludique, donc, comme simple fait de jouer, d'être « au jeu », comme le dit la joueuse agacée de notre bref dialogue. Qu'une notion si simple en surface puisse créer des malentendus comme celui que j'ai retranscrit est le signe qu'elle mérite d'être examinée plus avant. Je suivrai ici deux pistes complémentaires, celle de la normalisation sociale de l'action d'une part, et celle de la phénoménologie de l'expérience d'autre part.

3 Cette étude débouchera sur la présentation de MÉDIAL, un modèle multidimensionnel des formes de l'engagement, qui se propose d'articuler ces deux interrogations : qu'est-ce qu'être sous- ou sur-engagé dans un jeu? Et quelles sont les différentes manières de vivre l'engagement ludique? Ce modèle complexe vise à favoriser la prise en compte de ce 
qu'est le paysage ludique contemporain : multiplication des formes éditoriales hybrides, prolifération de jeux aux formats et supports très hétérogènes, constitution de communautés de pratique, de critique, voire de financement autour de segments précis du marché et émergence d'une culture ludologique partagée bien au-delà des cercles universitaires. Face à ces mutations, les typologies étanches à la manière de Roger Caillois, aussi populaires soient-elles dans les bibliographies, n'ont plus guère d'intérêt descriptif ${ }^{1}$.

\section{Engagement, distanciation et réflexivité}

4 La notion d'engagement peut s'étudier suivant plusieurs axes développés par les sciences sociales. Un premier axe, familier aux sociologues, oppose l'engagement à la « distanciation ». Pour Norbert Elias :

La vie sociale, telle que nous la connaissons, s'effondrerait si les normes du comportement allaient trop loin dans une direction ou dans l'autre. Pour être précis : la possibilité de toute vie de groupe ordonnée repose sur l'interaction, dans la pensée ou l'activité humaines, d'impulsions dont les unes tendent vers l'engagement et les autres vers la distanciation. (Elias, 1993, p. 10)

5 L'intérêt du propos d'Elias est d'insister sur l'équilibre dynamique entre les deux pôles qu'il dessine, et sur le risque induit par un excès, un basculement vers l'un ou vers l'autre. Dans le cas de la pratique ludique, ceci invite à considérer l'engagement comme un descripteur souple des différentes formes d'expérience que nous traversons et observons : je sais si je suis engagé dans un jeu, et je peux ressentir des penchants au surengagement, comme l'emprise exercée par un Tetris (Pajitnov, 1984) ou un jeu de casino, comme le risque d'un sous-engagement lorsque j'abandonne un jeu en cours de partie. Surtout, je constate que ces tendances ne sont pas homogènes : les autres joueurs placent différemment leur propre curseur de l'engagement, au point que cela peut engendrer des conflits si nous tentons de jouer ensemble.

Pour rester le plus inclusif possible, je définirai l'engagement comme une mobilisation socialement normée des participants d'une expérience, sous la forme de présence corporelle ou virtuelle, d'attention et de mémoire, de manifestation d'affects.

Dire que la mobilisation est "socialement normée » implique d'abord qu'il existe des risques de sous-engagement et de sur-engagement. Le sous-engagement est familier à tous les joueurs: l'un des participants enfreint l'un ou plusieurs des termes de ma définition par son défaut de présence, d'attention, de mémoire et/ou de mobilisation d'affects. Il n'écoute pas la règle, quitte régulièrement la table ou se laisse distraire par son téléphone, repose constamment les mêmes questions, perd le tempo des tours de jeu ("C'est à moi ? Déjà ? ), fait exprès de perdre pour se coucher plus tôt, joue de manière mécanique aux jeux d'ambiance ou de manière exubérante aux jeux de stratégie, empile, détruit ou disperse le matériel, etc.

8 Que fait-il donc dans le jeu, activité non-contrainte par excellence? Les joueurs sousengagés n'intéressent d'ordinaire pas les sociologues, mais ils méritent qu'on s'interroge - et qu'on les interroge - sur les motifs de leur présence. Dans la plupart des cas, ils se disent « otages » de la situation : « Je suis vraiment l'erreur de casting de la soirée, mais je rentre en voiture avec la famille X.»; «Ce n'est pas moi qui aime jouer, mais quand ta copine adore ça... ». D'autres sont là pour jouer, mais ont simplement choisi la mauvaise table: «J'aurais dû demander combien de temps ça durait.»; "Je déteste les jeux 
coopératifs : au bout d'un quart d'heure, on est tous à la botte de celui qui connaît la règle par cœur et on fait le pousse-pion. » Enfin, il y a ceux dont l'engagement s'interrompt ou s'effiloche en cours de partie, souvent par fatigue ou par déception d'une attente ludique précise : «Quatre mauvais jets de dés dans les premiers tours, j'ai plus aucune chance de gagner. » « "Je suis tombée sur des connards qui jouent ensemble depuis des années, il fallait que ça se termine vite».

Le sur-engagement se caractérise au contraire par un excès de la mobilisation décrite dans notre définition: avalanche de conseils ou d'ordres du joueur expert qui instrumentalise les "pousse-pion» dans le jeu coopératif évoqué ci-dessus, hyperconcentration du stratège qui passe quinze minutes à peaufiner chaque coup et rend la partie interminable, débordement émotionnel du vainqueur immodeste ou de l'imprécateur qui maudit ciel et terre après chaque coup. Poussé à l'extrême, le surengagement peut briser le cadre social de la partie, notamment dans des jeux de société fondés sur le risque de trahison comme Diplomacy (Calhamer, 1976). Dans Les Cadres de l'expérience, Erving Goffman donne plusieurs exemples de ce qu'il appelle les «sousmodalisations ", durant lesquelles des participants d'un jeu ou d'une rencontre sportive en viennent aux mains ou basculent dans une terreur irraisonnée (Goffman, 1991, p. 352-353).

Certaines tablées expriment leurs attentes d'engagement pour désamorcer tout risque de querelle: "on coupe les portables pendant la partie ", "on ne parle qu'à son tour ". D'autres gèrent les tensions à mesure qu'elles se manifestent, souvent par la mobilité des participants d'une partie à l'autre. On retrouve ces motifs de gestion collective de l'engagement bien au-delà des tables de jeu, que ce soit en ligne (Berry, 2012) ou en jeu de rôle grandeur nature (Kapp, 2013). Qu'est-ce qu'être « distrait par son téléphone », « trop peu attentif aux explications » ou « trop longtemps plongé dans la réflexion »? Le « trop peu » comme le « trop » ne se délimitent pas dans l'abstrait, mais au sein des collectifs de joueurs ${ }^{2}$, où l'on voit s'entremêler des déterminants culturels (le fait de parler ou de faire silence en cours de partie varie fortement d'un pays à l'autre) et des profils personnels (on sait que telle joueuse a besoin de temps pour préparer ses coups, que tel joueur est colérique, etc.). C'est le social qui trace des limites haute et basse de l'engagement, pas le sociologue.

11 Une autre lecture de l'engagement ludique consiste à y voir une pratique exclusive et focalisée, qui ne laisserait aucune part au retour réflexif. Sur ce second axe " engagement-réflexivité », on retrouve en filigrane l'idée d'une distanciation, puisque la réflexivité est présentée comme une prise de recul, qui devrait se manifester dans une temporalité distincte de celle du jeu. Ceci ne correspond pas à ce qu'on observe, tant dans la pratique des jeux vidéo qu'autour des tables. Il suffit de suivre des séquences vidéoludiques commentées en direct par les joueurs ou d'assister à une partie de jeu de rôle pour recueillir toutes sortes de manifestations de réflexivité. Pour Kendall L. Walton :

Parmi les œuvres qui limitent notre engagement dans les mondes fictionnels figurent des chefs-d'œuvre reconnus, qui peuvent être à leur manière hautement provocateurs, dépaysants, convaincants. (Walton, 1990, p. 274)

Les manifestations de réflexivité peuvent, certes, diminuer le sentiment d'immersion dans une diégèse fictionnelle (Caïra, 2014), mais elles n'affectent pas le phénomène plus global d'engagement ludique. Pourquoi les concepteurs et éditeurs de jeux encourageraient-ils autant la réflexivité de leurs joueurs, par toutes sortes de références et de traits d'humour, si elle était synonyme de distanciation? J'estime au contraire que 
la réflexivité est une dimension à part entière de l'engagement, et qu'elle doit par conséquent faire partie intégrante des modèles de l'expérience ludique développés par les sciences du jeu.

\section{Du flow et des raisons de l'endiguer} évoquer le concept de flow théorisé par le psychologue Mihály Csíkszentmihályi. Ses livres et articles sont régulièrement cités dans les revues telles que Game Studies et Sciences du $j e u$, notamment dans les appels à contribution, ce qui est un bon indicateur de l'influence d'un concept ou d'un auteur. Rappelons l'une des définitions les plus détaillées qu'en donne le chercheur hongrois :

Premièrement, l'expérience se produit d'ordinaire quand nous nous confrontons à des tâches que nous avons des chances d'accomplir. Deuxièmement, nous devons pouvoir nous concentrer sur ce que nous faisons. Troisièmement et quatrièmement, la concentration est généralement possible parce que la tâche entreprise présente des objectifs clairs et nous procure un feed-back immédiat. Cinquièmement, l'on agit avec une implication profonde mais naturelle, qui libère la conscience des inquiétudes et des frustrations de la vie quotidienne. Sixièmement, des expériences agréables permettent aux gens d'éprouver un sentiment de contrôle sur leurs actions. Septièmement, le souci de soi disparaît, mais paradoxalement la conscience de soi apparaît plus forte une fois l'expérience du flow terminée. Enfin, le sens de la durée est altéré ; les heures passent comme des minutes et les minutes peuvent sembler durer des heures. La combinaison de tous ces éléments procure une profonde jouissance, si satisfaisante que les gens estiment qu'elle vaut la peine d'y consacrer une énergie importante. (Csíkszentmihályi, 2008, p. 49, ma traduction)

traits de la notion de flow pour les sciences du jeu apparaissent dans cette longue citation. La définition est fondée sur des critères précis qui permettent des validations empiriques (notamment Weibel et Wissmath, 2011). Csíkszentmihályi met en avant les caractéristiques saillantes des activités autotéliques, dont le jeu fait partie. L'auteur séduit ainsi par sa prise en compte de la difficulté, de l'apprentissage et du plaisir dans l'expérience du jeu. Sa description des temps forts du jeu et d'un rapport au temps altéré est très proche du ressenti exprimé par de nombreux joueurs dans les enquêtes de terrain ou lors de discussions spontanées. On comprend donc le succès que peut connaître le concept de flow dans la ludologie, particulièrement dans l'étude du jeu numérique. Jesper Juul parle ainsi d'une "parfaite correspondance» (Juul, 2005, p. 112) entre le cadre théorique de Csíkszentmihályi et l'idée d'ajustement dynamique de la difficulté (Dynamic Difficulty Adjustment). En dialogue avec Geoffrey M. Rockwell sur les serious games, Kevin Kee (2011) cite le flow comme manière de réconcilier plaisir et travail. Dans leur article sur l'appréciation de l'expérience vidéoludique, Maxime Deslongchamps-Gagnon et Alexandre Poirier (2016) reprennent également l'idée de flow comme «harmonie dans l'interaction défi-compétence ». La popularité du flow étant contemporaine des grands débats sur l'immersion, les deux concepts ont pu se favoriser mutuellement par leur complémentarité. Le flow peut en effet apparaître comme une expérience de l'engagement dans l'épreuve qui s'articule avec de celle d'immersion dans une diégèse. Csíkszentmihályi associant flow et jouissance, sa description de l'expérience optimale reprise par des concepteurs de jeu comme Jenova Chen (2007) devient clairement prescriptive: si le flow procure du plaisir, il devient souhaitable de créer des jeux qui suscitent le flow. Cette naturalisation d'une forme réputée parfaite de l'engagement 
ludique, donc du game design comme recherche d'une sorte de «point $\mathrm{G}$ » du joueur, a suscité des commentaires critiques, dont celui de Greg Costikyan :

On consacre beaucoup de temps et d'énergie à essayer de créer des jeux qui induisent un «état de flow ». Bien que cela soit vrai pour certains jeux, je dirais que c'est loin d'être le cas pour tous - et que beaucoup de jeux profitent précisément du fait de tenir le joueur éloigné de tout sentiment de flow. (Costikyan, 2013, p. 25, ma traduction)

15 J'abonde dans le sens de Costikyan au simple examen de l'offre contemporaine de jeux et des usages observés sur le terrain. Mais j'ai surtout trois objections plus fondamentales contre l'idée d'expérience optimale dans le jeu. La première critique porte sur les apprentissages. On présente la compétence du joueur comme non problématique, en s'appuyant notamment sur la notion de « courbe d'apprentissage ». Or il faut rappeler que cette courbe, historiquement liée à l'étude de la progression des sujets face à des tâches simples et répétitives, pose d'importants problèmes de méthode de mesure et d'applicabilité au jeu. L'idée de placer l'expérience en abscisses et l'apprentissage en ordonnées semble aller de soi, mais comment mesure-t-on l'expérience, si ce n'est par le temps passé à jouer, et l'apprentissage, si ce n'est par les performances du joueur? On rencontre un problème immédiat de mesure de ce qu'on essaie de représenter, mais également de théorie de l'apprentissage : est-il bien certain que tous les jeux créent un « apprentissage par la pratique » (learning-by-doing) ? Le sujet dépasse le périmètre de cet article, mais il mérite d'être rappelé : caler la difficulté d'un jeu sur la prétendue « courbe d'apprentissage » du joueur ne fonctionne qu'avec des tâches clairement identifiées, dans un contexte de répétition que peu de jeux contemporains favorisent (notamment parce qu'ils sont scénarisés ou paramétrés pour modifier l'expérience à chaque partie, et parce que les adversaires eux-mêmes changent et apprennent).

Ma seconde objection porte sur la régulation sociale du jeu. Le flow est une notion psychologique qui fait largement l'impasse sur le collectif: la plupart des situations décrites comme optimales par Csíkszentmihályi et ses émules reposent soit sur du jeu en solitaire, soit sur du jeu stratégique où les interactions avec l'adversaire sont limitées, comme les échecs pratiqués dans un cadre compétitif. Les témoignages de flow que j'ai pu recueillir au fil de mes travaux sont du même type : plongé dans un sudoku, il manque son arrêt de train; lancée dans un jeu vidéo, elle découvre qu'elle a passé une nuit blanche; etc. Si des groupes peuvent jouer très longtemps, notamment dans la pratique des jeux de rôle sur table ou grandeur nature, la focalisation sur une tâche particulière et la perte des repères temporels décrites par les psychologues y sont très rares. Est-ce à dire que les autres joueurs sont un facteur de perturbation du flow, de «non-optimalité " de l'expérience? Probablement. Et cela me semble être un enseignement sur le jeu de société : jouer à plusieurs, c'est accepter ce caractère non-optimal de la partie. Ce n'est pas une expérience « meilleure » ou « moins bonne » qu'un jeu solitaire qui tendrait vers le flow, simplement un autre aspect du vécu des joueurs. De même, autour des tables, le flow d'un seul joueur " optimalement » plongé dans le jeu apparait souvent comme forme de sur-engagement pénible pour le reste du groupe. Il n'y a aucune raison, ni théorique, ni empirique, de penser le jeu en général, et particulièrement le jeu de société, en termes d'expérience prétendument « optimale ».

17 Ma troisième critique est d'ordre épistémique. Grâce aux travaux de Csíkszentmihályi, on sait identifier, compter et mesurer les moments de flow dans de nombreux champs de l'expérience humaine. Je ne peux ni ne veux invalider la théorie du flow dans la pratique 
des jeux, puisque j'en ai des témoignages et des expériences de première main. L'arbre existe donc, solide et majestueux, mais quid de la forêt qu'il cache? Quid du reste de l'expérience, si l'on s'en tient à une posture d'observateur? À un niveau plus théorique, quid du «cadre paradoxal» du jeu (Bateson, 1977)? Comme l'expliquait Bateson, l'expérience ludique doit sa particularité à l'échange, dans un même cadre communicationnel, de messages contradictoires, tels que «Je t'attaque» et «Je ne t'attaque pas» dans le cas des jeux de confrontation. Ce cadre crée constamment la possibilité d'une distanciation du type "Ce n'est qu'un jeu", tout en requérant un engagement bien réel. Bateson décrit ainsi des dynamiques ludiques où la baisse de l'engagement révèle une posture en surplomb de l'action qui permet de revenir au jeu avec plus d'intensité. Or le flow ne tient absolument pas compte de cette logique du paradoxe, puisqu'il fonctionne purement "au premier degré », sur un seul niveau de mobilisation cognitive qui - du moins si l'on lit les exemples de Csíkszentmihályi - exclut la possibilité d'un recul réflexif en cours d'action.

Il ne s'agit donc pas de nier ni la pertinence du concept de flow, ni son intérêt en tant qu'outil descriptif, mais de circonscrire son champ d'application dans les sciences du jeu. Il fonctionne à merveille pour certaines expériences paroxystiques du jeu, notamment dans des contextes compétitifs exacerbés, mais ne doit pas oblitérer les autres dimensions de l'expérience. S'il en partage de nombreux traits structuraux, le jeu n'est pas un sport de compétition. Dans le jeu de société, la dimension compétitive s'accompagne d'autres formes d'engagement qui ne sont pas des impuretés à éliminer. Le jeu de société «fait société ", rassemble les participants autour de la table, en tant qu'expérience nonoptimale : ses scories peuvent être une force.

\section{MÉDIAL}

Parmi les chercheurs qui tentent de penser la pluralité des formes d'engagement, Gordon Calleja (2011) a fourni l'un des modèles les plus stimulants. Issu de son enquête sur la pratique des jeux vidéo, il se révèle généralisable à d'autres formes d'expériences ludiques. Calleja propose d'abord une distinction entre macro- et micro-engagement. Si la dimension « micro- » correspond au temps de jeu proprement dit, le « macro- » intègre des formes plus diffuses et négligées de la relation au jeu: test, achat et prescription d'achat, lecture et production de critiques, etc. Ces deux niveaux d'étude enrichissent les méthodes d'enquête. Ils permettent d'appréhender la globalité du rapport des acteurs sociaux aux jeux qu'ils pratiquent, mais également à ceux qu'ils refusent de pratiquer ou qu'ils aimeraient pratiquer.

La diversité des formes d'engagement, telle que la présente Calleja, fait apparaître le jeu comme intrinsèquement complexe et non-optimal. Il est possible de s'engager dans six directions : kinesthésique, spatiale, partagée, narrative, affective et ludique (2011, p. 38 ; la figure 1 a été modifiée). Loin du caractère excluant des approches par le flow, son modèle encourage les chercheurs à prendre en compte des jeux peu propices à l'immersion spatiale (qu'il appelle "immersion as transportation ») et à la mise à l'épreuve dans un système abstrait (« immersion as absorption »). 


\section{L'engagement ludique selon Calleja (2011)}

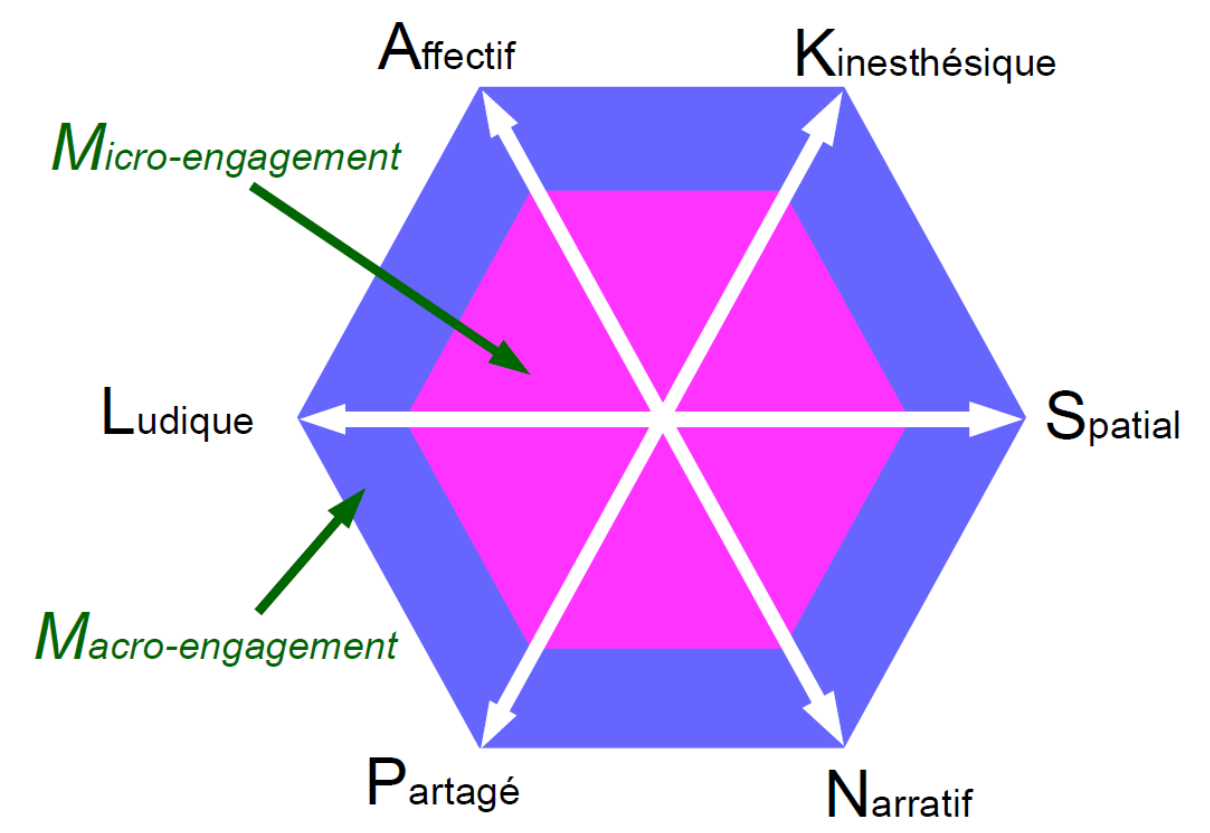

La place manque pour faire un rappel exhaustif des définitions de Calleja sur chacune des directions de l'engagement qu'il propose. L'essentiel à retenir, me semble-t-il, est l'idée que ces flèches pointent dans des directions différentes, et qu'un engagement fort dans une des dimensions de l'expérience ludique se fait le plus souvent au détriment des autres dimensions. Difficile, par exemple, de créer une forte tension sur l'intrigue alors que le joueur est occupé à prendre en main l'interface d'un jeu, d'où le caractère narrativement "faible " de nombreuses phases tutorielles dans les jeux vidéo (le personnage est à l'entrainement ou mène une mission de routine, son environnement est stable, ses éventuelles erreurs sont sans conséquence). On ne saurait par conséquent atteindre un état stable d'engagement optimal que dans des jeux en solitaire au contenu expérientiel très homogène, typiquement les casse-têtes ou les jeux vidéo de course automobile. On constate que, dans la majorité des jeux contemporains, la dynamique de la partie crée des sollicitations diverses dans les dimensions multiples du modèle. Calleja invite donc à penser le jeu comme intrinsèquement composite.

Le modèle MEDIAL, que je présente ici, incorpore à la structure proposée par Calleja les différentes thèses défendues plus haut. Les termes « micro- » et « macro-engagement » ne désignant pas tant le type d'engagement que le découpage du temps, je propose de parler simplement d'une expérience «En-partie » incluse dans un vécu plus large de joueur «Hors-partie». J'ajoute une épaisseur au schéma pour faire apparaître la notion d'intensité de l'engagement: en surface se trouve le sous-engagement, en profondeur le sur-engagement. Il est tout à fait possible de faire preuve de sur-engagement hors-partie, c'en est même un symptôme assez fort.

Figure 2 


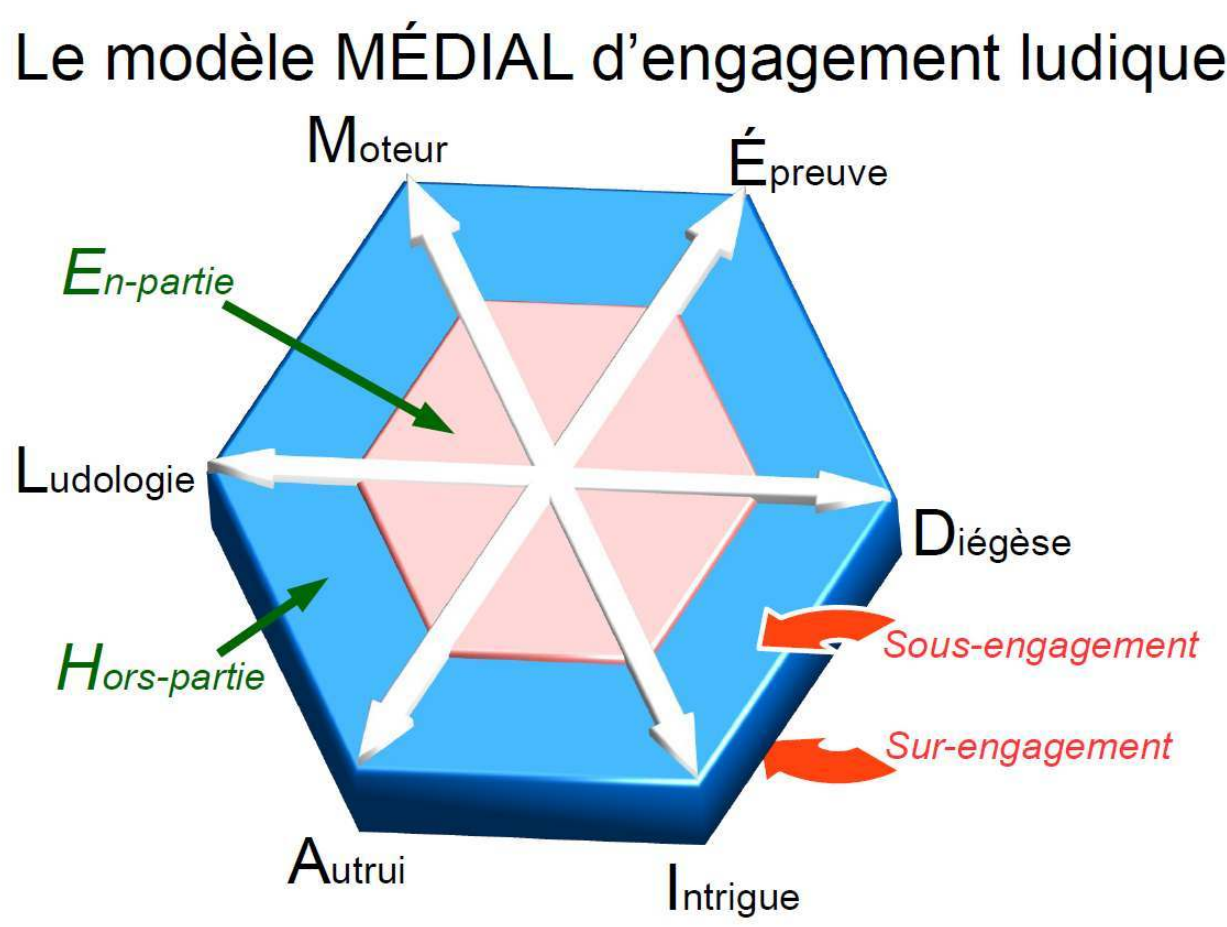

Si je conserve la forme hexagonale, il me semble que la « flèche » de l'engagement affectif n'en est pas une. Cela ne signifie pas que les affects n'interviennent pas, mais bien au contraire qu'ils sont partout dans l'expérience ludique, qu'on se place du point de vue de la production ou de celui de la réception. En effet, les manifestations d'affects ne me semblent pas représenter une dimension à part entière, ni du game design ni de l'expérience du joueur, au sens où l'on ne peut pas les séparer des autres "flèches » de Calleja : telle émotion sera rattachée à une trouvaille narrative, telle autre à la découverte spatiale, une troisième au caractère partagé du jeu, etc. De même, dans un studio, personne ne peut développer la question affective comme une dimension distincte du travail des scénaristes ou des concepteurs de niveau (level designers). J'intègre donc l'affectif aux autres formes de l'engagement. En revanche, une autre sixième flèche me semble nécessaire: celle de l'expérience ludologique. Il s'agit de rendre raison d'un engagement métaludique, comme Roman Jakobson (1963) faisait apparaître le métalinguistique comme une fonction du langage à part entière. Les six directions retenues pour étudier l'engagement des joueurs sont donc les suivantes :

Moteur: Cette première direction désigne l'intérêt porté au système de règles, à la « mécanique » logico-mathématique ou physique qui régit le jeu. On note dès l'explication des règles autour d'une table ou dès la prise en main d'un jeu vidéo des comportements ou des questions qui visent à explorer les potentialités et les limites de ce système. La volonté de comprendre le moteur peut être liée à un engagement fort dans la dimension suivante, celle de l'épreuve, mais ce n'est pas nécessairement le cas : on voit ainsi certains joueurs expérimentés tester des stratégies inédites, non dans l'optique de gagner, mais dans celle de "savoir si on peut aussi gagner comme ça». De même, l'attrait des joueurs passionnés pour les extensions et variantes révèle une volonté de découvrir de nouvelles facettes du moteur, pas des manières de gagner plus sûrement.

Épreuve: On est ici au plus proche de la notion de flow, puisqu'on étudie dans cette direction la manière dont les joueurs mobilisent leurs moyens intellectuels et physiques 
pour tenter de gagner la partie ou, si le jeu n'explicite pas de conditions de victoire, de produire la meilleure performance possible. L'engagement hors-partie recouvre toutes les manières de s'entraîner dans le but de jouer mieux: étudier les probabilités pour le poker, noter du vocabulaire ou des conjugaisons inusitées pour le Scrabble, etc. Le surengagement consiste à chercher à gagner par tous les moyens, y compris ceux qui détruisent l'engagement des autres joueurs.

Diégèse : Cette forme de l'engagement n'est bien entendu développée que dans la vaste famille des jeux de simulation, que l'on peut définir brièvement par l'articulation entre un « système » et un «thème » ou, dans les termes du présent modèle, un moteur et une diégèse. Difficile, en effet, de s'engager dans la diégèse - encore moins dans le "monde " - d'un jeu abstrait comme le go, le craps, le billard carambole ou le 2048 (Cirulli, 2014). Au contraire, de nombreux développeurs de jeux ont misé sur la thématisation, sur le worldbuilding et sur une forte promesse d'immersion pour renforcer l'attrait de leurs productions. L'engagement diégétique commence par l'adoption du vocabulaire du jeu : dire «Bois et Or » et non «marron et jaune » à Kingsburg, par exemple. Hors-partie, il peut engendrer des lectures sur le genre ou l'œuvre adaptée, voire des loisirs créatifs comme on en observe fréquemment en jeu de rôle.

Intrigue : La narrativité des jeux peut s'entendre au sens fort d'une scénarisation explicite de chaque partie, ou au sens plus diffus d'une capacité des joueurs à produire des récits de parties comme suite d'événements intra-diégétiques liés par certaines relations causales. La scénarisation est l'une des tendances fortes de la production actuelle, qu'il s'agisse du jeu de plateau ou du jeu vidéo. Il ne s'agit donc plus d'opposer narratologues et ludologues, mais de travailler sur les complémentarités et les échanges possibles entre leurs disciplines. Passer de la notion d'engagement narratif chez Calleja à celle d'engagement par l'intrigue dans MÉDIAL n'est pas neutre. Nombre de jeux ne sont pas scénarisés mais créent une véritable intrigue, une tension narrative sur les événements au sein de leur diégèse qui correspond aux enjeux vécus au niveau extradiégétique par le(s) joueur(s) (Baroni, 2007).

27 Autrui : Créer des interactions sociales, voire nouer des relations, peut être un objectif en soi ou un simple effet induit de l'engagement ludique. Les enquêtes sur le jeu de rôle en ligne montrent bien que la socialisation renforce l'engagement sur la durée des joueurs, même lorsque ceux-ci estiment avoir « fait le tour » du jeu. La dimension " autrui » d'un jeu est un vaste domaine d'enquête, qui se déploie aux deux niveaux intra- et extradiégétique : savoir que son voisin de table joue Catwoman, fût-ce le temps d'une partie, crée une vaste palette de possibilités d'interaction entre joueurs, entre personnages, voire d'un joueur au personnage de l'autre (« La prochaine fois, choisis mieux ton joueur, Catwoman!»). Cet engagement peut concerner autant les personnages des joueurs que des personnages non-joueurs animés par un meneur de jeu ou un logiciel. Rien ne permet de tracer a priori la limite des relations qui vont engendrer de l'interaction ludique : les êtres de fiction créés pour les jeux peuvent créer des liens avec les joueurs, de même que certains personnages romanesques ou cinématographiques occupent une position centrale dans notre univers affectif.

28 Ludologie : C'est la dimension qui, à mes yeux, manque le plus au modèle de Calleja. Sur table ou face à un écran, en solitaire ou en pratique de masse, les joueurs passionnés développent une pensée réflexive et de plus en plus érudite. Cette nouvelle direction s'intègre à la pensée multidirectionnelle promue par Calleja, puisque le discours ludologique en cours de partie traduit bien la forte implication du joueur, mais peut 
facilement contrecarrer les autres formes de l'engagement. Lorsqu'un joueur manque un test de négociation lors d'une session de jeu de rôle médiéval, et commente : "Je viens d'acheter un Bois pour quatre Moutons ", la plupart des convives saisissent la référence aux Colons de Catane (Teuber, 1995), qui fera sourire les uns mais pourra contrarier la dynamique d'immersion des autres. La réflexivité ne s'arrête pas à ces clins d'œil : elle inclut de plus en plus de remarques sur les parentés entre mécanismes ou entre thèmes, sur les choix éditoriaux, sur les logiques économiques ou sur les grandes " écoles » de développement de jeux. De grands débats entre joueurs, notamment sur la légitimité du financement participatif, sur l'addiction au jeu ou sur l'existence de traditions nationales de game design, ont contribué à diffuser cette culture réflexive, et de plus en plus d'organisateurs de conventions inscrivent des cycles de conférences à leur programme. Les sciences du jeu ne peuvent plus s'exclure elles-mêmes de leur objet d'étude.

\section{Conclusion}

La partie de Kingsburg s'achève sur une victoire de la joueuse qui a expliqué les règles. Son voisin se lève avec un bref « Pas un jeu pour moi, ça. » et rejoint un groupe lancé dans des party games. Les autres joueurs demandent à faire une seconde partie avec l'extension «Forgez un royaume». Tout en préparant cette nouvelle partie, ils cherchent des équivalents du système de ce jeu en termes de placement de dés sur des cases de ressources. Sur le modèle MÉDIAL, ce groupe choisit d'approfondir son engagement dans le Moteur. Le joueur qui les quitte ne sort pas du modèle, mais s'y déplace, probablement vers l'Autrui, si c'est un jeu d'ambiance qui a sa préférence.

MÉDIAL permet de cartographier les jeux et les joueurs sur un espace complexe, sans opposer la table à l'écran et sans préjuger des formes « souhaitables » de l'engagement ${ }^{3}$. Il peut servir à étudier la production, car le game design ne se résume pas à la recherche d'un état optimal, mais suppose une composition parmi diverses formes possibles d'implication (sociale, tactique, narrative...) et un choix de leur intensité. Enfin, MÉDIAL vise à faire exister les postures métaludiques du chercheur et du développeur dans un modèle d'engagement, donc à ne pas faire de la recherche en sciences du jeu une démarche aveugle à elle-même.

Admettre des variations d'engagement, c'est aussi réhabiliter les temps faibles de l'expérience ludique, et par conséquent de penser les temps forts pour ce qu'ils sont: rares, intenses, marquants. Il ne s'agit pas de tourner le dos à plusieurs années de discussion sur le flow, mais de repenser le périmètre d'application de ce concept. Le flow est une des clés de l'expérience ludique, mais l'erreur serait de considérer qu'elle ouvre toutes les portes.

\section{BIBLIOGRAPHIE}

BATESON G. (1977), Vers une écologie de l'esprit T.1, Paris, Éditions du Seuil. 
BERRY V. (2012), L'expérience virtuelle, Rennes, Presses universitaires de Rennes.

CAÏRA O. (2007), Jeux de rôle, les forges de la fiction, Paris, CNRS Editions.

CAÏRA O. (2014), «L'Expérience fictionnelle, de l'engagement à l'immersion », in B. Guelton (dir.), Les Figures de l'immersion, Rennes, Presses universitaires de Rennes, pp. 61- 76.

CALLEJA G. (2011), In-Game, Cambridge, MIT Press.

CHEN J. (2007), Flow in Games, MFA Thesis, www.jenovachen.com

COSTIKYAN G. (2013), Uncertainty in Games, Cambridge, MIT Press.

CSÍKSZENTMIHÁLYI M. (2008), Flow, The Psychology of Optimal Experience, New York, Harper Perennial Modern Classics.

DESLONGCHAMPS-GAGNON M. et POIRIER A. (2016), « L'expérience émotionnelle du joueur en échec dans l'art vidéoludique ", Sciences du jeu n 6, https://journals.openedition.org/sdj/718.

ELIAS N. (1993), Engagement et Distanciation, Paris, Fayard.

GOFFMAN E. (1991), Les cadres de l'expérience, Paris, Éditions de Minuit.

JAKOBSON R. (1963), Essais de linguistique générale (t.1), Paris, Éditions de Minuit.

JUUL J. (2005), Half-Real, Cambridge, MIT Press.

KAPP S. (2013), L'immersion fictionnelle collaborative, Une étude de la posture d'engagement dans les jeux de rôles grandeur nature, thèse de doctorat, EHESS/Université Libre de Bruxelles.

ROCKWELL G. M. et KEE K. (2011), « The Leisure of Serious Games : A Dialogue », Game Studies n -11, vol. 2, http://gamestudies.org/1102/articles/geoffrey_rockwell_kevin_kee

WALTON K. (1990), Mimesis as Make-Believe, Cambridge, Harvard University Press.

WEIBEL D. et WISSMATH B. (2011), « Immersion in Computer Games: The Role of Spatial Presence and Flow », International Journal of Computer Games Technology, vol. 2011, Article ID 282345, https:// www.hindawi.com/journals/ijcgt/2011/282345/

\section{NOTES}

1. L'analyse de l'engagement ludique qui suit découle d'une part de nombreuses observations, le plus souvent participantes, que j'ai pu mener sur différents terrains : jeu de rôle sur table, jeux de cartes et de plateau, jeux vidéo, storygames, etc. Elle s'enrichit depuis une dizaine d'années d'une activité de développement de mes propres jeux et de scénarisation sur les créations existantes, qui m'a permis de prendre en compte de nouvelles situations: alpha- et bêta-tests sur des prototypes, présentation à des éditeurs, travail de "rethématisation » de certains jeux, et plus récemment réflexions sur l'adaptabilité de certaines mécaniques de jeu à d'autres supports. Cette double approche des objets est tout à fait compatible avec la sociologie d'inspiration goffmanienne que je pratique pour étudier les expériences de la fiction et du jeu. La notion de cadre social étant centrale dans mon rapport au terrain, la pluralité des situations et des rôles qu'on peut y jouer éclaire davantage les objets d'étude que ce que la seule «casquette » de l'enquêteur me permettait d'observer autrefois.

2. Cette normalisation sociale s'exerce moins, voire pas du tout, sur le joueur solitaire. Son sousengagement est indolore, et son sur-engagement ne se paie que s'il se répercute dans le reste de son quotidien: tensions avec l'entourage, perturbation des cycles du sommeil et/ou de l'alimentation, irritabilité, pertes financières, etc. C'est pourquoi la sociologie des expériences 
ludiques en solitaire pose des problèmes cruciaux de généralisation, comme on va le voir dans la section dédiée au concept du flow.

3. Cette démarche reprend et généralise la cartographie des attentes ludiques que j'avais proposée pour l'analyse des jeux de rôle sur table (Caïra 2007).

\section{RÉSUMÉS}

Une définition en vogue de l'engagement est celle du flow, théorisée par M. Csíkszentmihályi. Elle présente un grand intérêt pour les sciences du jeu, notamment par la mise en avant d'activités autotéliques et par la prise en compte du défi, de l'apprentissage et du plaisir. Néanmoins, cette seule clé n'ouvre pas toutes les portes. Un modèle comme celui de G. Calleja semble plus adapté à l'étude des jeux contemporains. Les six dimensions qu'il explore font apparaître le jeu comme intrinsèquement complexe et non optimal. Sa distinction entre macro- et micro-engagement révèle des formes négligées de la relation au jeu qui se manifestent en dehors des parties. Le modèle MÉDIAL (Moteur, Épreuve, Diégèse, Intrigue, Autrui et Ludologie) reprend l'approche multidimensionnelle de Calleja en y ajoutant deux ambitions. Premièrement, le sous- et le surengagement y apparaissent comme des formes socialement problématiques de l'expérience ludique. Deuxièmement, la réflexivité n'est plus traitée comme une distanciation mais considérée comme une dimension à part entière de l'engagement. MÉDIAL permet de cartographier les jeux et les joueurs dans un espace complexe, sans préjuger de la légitimité des formes de l'engagement.

M. Csíkszentmihályi's theory of flow provides a popular definition of engagement in games. It is of great interest for game studies, particularly by its emphasis on autotelic activities and by its consideration of challenge, learning and pleasure. However, flow cannot provide all the keys to engagement in games. G. Calleja's model seems more suited for the study of contemporary games. The six dimensions he explores reveal gaming as an intrinsically complex and non optimal behaviour. His distinction between macro- and micro-engagement sheds light on neglected forms of our relationship to games, which manifest themselves outside gaming sessions. The MÉDIAL model (Moteur [Engine], Épreuve [Test], Diégèse [Diegesis], Intrigue [Plot], Autrui [Others] and Ludologie [Ludology]) takes up Calleja's multidimensional approach, adding two ambitions to it. Firstly, under- and over-engagement must appear as socially problematic behaviours. Secondly, reflexivity must not be treated as a form of detachment but as a genuine dimension of engagement in games. MÉDIAL allows mapping games and gamers within a complex space and without prejudging the legitimacy of each form of engagement.

\section{INDEX}

Keywords : engagement, under-engagement, over-engagement, social norms, Flow, reflexivity, ludology

Mots-clés : engagement, sous-engagement, sur-engagement, normes sociales, Flow, réflexivité, ludologie 


\section{AUTEUR}

\section{OLIVIER CAÏRA}

Centre Pierre Naville - Université Paris Saclay 